\title{
6.10 Bibliometrische Dienstleistungen
}

\section{Einleitung}

Forschungs- und Wissenschafts-Rankings sind populär. Es gibt keinen Universitätspräsidenten, der nicht die Position seiner Einrichtung im nationalen und internationalen Vergleich kennt (und sie erklären oder instrumentalisieren kann), und kein Politiker kommt ohne sie aus: „Wissenschaft und Politik werden sich gegenseitig beeinflussen und beobachten"1. Bibliometrie wird heute zunehmend verknüpft mit Leistungsmessung, Leistungsbewertung, Evaluation und wissenschaftspolitischer Steuerung.

Für die Auswertung des wissenschaftlichen Outputs und ihren Einfluss auf die Entwicklung eines Fachgebiets werden grundsätzlich zwei Methoden eingesetzt, eine qualitative und eine quantitative. $\mathrm{Zu}$ einen ist dies die wissenschaftliche Begutachtung (peer review) und zum anderen die bibliometrische Analyse.

Die Peer-Review-Methode kann im Unterschied zu bibliometrischen Methoden kaum durch objektive Merkmale bestimmt werden. Es handelt sich dabei um Einschätzungen kompetenter Fachexperten, die oft als subjektiv bezeichnet werden. Zumindest lässt sie sich nicht quantifizieren.

Bibliometrische Analysen hingegen gewähren Einsicht in alle wichtigen Komponenten der Wissenschaft auf der Ebene von Makroforschungen: die Struktur wissenschaftlicher Aktivitäten in einzelnen Disziplinen auf nationaler Ebene, die wissenschaftliche Produktivität aufgeschlüsselt nach Ländern, der Einfluss einzelner Länder oder Regionen auf bestimmte Wissensgebiete, internationale und regionale Zusammenarbeit, die Informiertheit von Wissenschaftlern über Entwicklungen einzelner Wissenschaftsbereiche in der unmittelbaren oder weiteren Umgebung, die Benutzung formaler Kommunikationskanäle in einem Land, das wissenschaftliche Produkt und sein Einfluss, institutionelle Zusammenarbeit usw. Objekte dieser Forschungen sind Produzenten von Publikationen (individuelle Autoren, Teams, Einrichtungen, Länder und Regionen), die Publikationen selbst (Zeitschriften, Artikel, sekundäre Informationsquellen) sowie deren deskriptive Eigenschaften und Zitatenanalysen, die wissenschaftliche Kommunikationsprozesse erschließen. ${ }^{2}$

Der moderne Begriff von Bibliometrie ist noch sehr jung. Er wurde 1969 von Pritchard als „statistical bibliography“ geprägt ${ }^{3}$, während bereits 1934 der Begriff in einem anderen Zusammenhang gebraucht wurde.

1 Taubert 2013.

2 Moed [u.a.] 1992.

3 Pritchard 1969: 348-349. „Therefore it is suggested that a better name for this subject [statistical bibliography] is bibliometrics, i.e. the application of mathematics and statistical methods to books and other media of communication." 
Gorraiz definiert sie heute als „Anwendung mathematischer und statistischer Methoden zur Erklärung der Prozesse der schriftlichen Mitteilungen“"4.

Die Inhalte von Bibliometrie sind dagegen viel älter. Allerdings in einer Anwendung, wie sie heute kaum noch existiert: als bibliographische Statistik zur Untersuchung von Veröffentlichungen $\mathrm{zu}$ speziellen thematischen Fragestellungen. Die Ergebnisse dieser Untersuchungen dienten dabei ausschließlich der inhaltlichen Bewertung zu konkreten Fragestellungen nach Sachthemen. Damit zeigt sich, dass die eigentliche und ursprüngliche Anwendung von Bibliometrie eher eine bibliothekarische war.

\section{Grundlagen bibliometrischer Analysen}

\subsection{Outputanalyse}

Die Zählung des wissenschaftlichen Outputs einer Person, einer Organisation, einer Region oder eines Staates bzw. von Staatengruppen erfolgt durch die Bestimmung der Menge an wissenschaftlichen Veröffentlichungen. Dies ergibt schon einen ersten Hinweis auf die Produktivität etwa eines Wissenschaftlers oder Staates. Allerdings sind hierbei noch keine qualitativen Aspekte berücksichtigt. Wer viel publiziert, muss deswegen noch kein guter Wissenschaftler sein oder besser als ein Wissenschaftler, der weniger veröffentlicht. Die Zahl der Publikationen sagt als direkter Indikator noch wenig aus, wenn er nicht in Bezug gesetzt wird zu anderen Größen.

Gleichwohl ist die Messung der Anzahl der veröffentlichten Einheiten die Grundlage für alle weiteren, sogenannten indirekten bibliometrischen Indikatoren. Dabei ist es nicht einfach, diese Grundzahl zu ermitteln. Woher sie kommt, ist auch eine Frage der Datenbasis, die verwendet wird.

Hierbei ist es besonders wichtig, Datenbasis und Art des wissenschaftlichen Outputs zu benennen. Am wichtigsten sind dabei Veröffentlichungen in Zeitschriften und Konferenzbänden, Bücher und Vorträge. Die Veränderung der Wissenschaftskommunikation hat allerdings zu einer größeren Vielfalt der Veröffentlichungsarten geführt, so dass durchaus auch Einträge in (wissenschaftlichen) Blogs und Chats, Twitterkanäle, YouTube und andere (soziale) Netzwerke relevanten, berücksichtigungswürdigen wissenschaftlichen Output bedeuten können.

4 Gorraiz 2004. 


\subsection{Resonanzanalyse (Wahrnehmungsanalyse)}

Die Resonanzanalyse ist eine höhere Form der bibliometrischen Analyse. Hierbei wird nicht einfach die Anzahl von Veröffentlichungen einer Person, einer Organisation oder einer Region bestimmt, sondern deren Wahrnehmung in der Scientific community. Wie aber wird Wahrnehmung bestimmbar?

Das wissenschaftliche System der Erkenntnisgewinnung basiert auf dem schrittweisen Hinzufügen neuer Erkenntnisse zu bereits vorhandenen. In kritischer Würdigung dessen, was andere bereits geleistet und veröffentlicht haben, werden einer Fragestellung neue Aspekte hinzugefügt oder Bestehendes korrigiert. Immer jedoch bezieht sich ein Wissenschaftler auf den aktuellen Status quo, den er berücksichtigt und zitiert. Mit diesem System, das die Leistungen anderer Wissenschaftler anerkennt oder sich kritisch mit ihnen auseinandersetzt, aber immer benennt, entsteht eine bestimmte Wahrnehmungsgeschichte von Ideen und Erkenntnissen, die man auch Rezeptionsgeschichte nennen könnte. Aber anders als der Begriff der Rezeptionsgeschichte in Literatur und Geschichte verstanden wird, bedeutet er in der Bibliometrie die Messung der Resonanz, also die Messung der Häufigkeit von Zitaten bestimmter Publikationen durch andere Wissenschaftler. Dies ist der wichtigste Basisindikator der Resonanzanalyse. Die Zitierhäufigkeit beschreibt dabei nur die Anzahl der Zitierungen einer bestimmten Publikation in einem festgelegten Zeitraum. Das Maß der Zitierhäufigkeit ist eine einfache Zahl, etwa 105. Sie bedeutet, die Publikation A ist im Zeitraum B 105 Mal zitiert worden.

Für die Anwendung der Wahrnehmungsanalyse muss man allerdings voraussetzen, dass die Anzahl der Zitierungen ein Indiz für die Qualität einer wissenschaftlichen Arbeit ist.

Kann man dieser Annahme nicht folgen (und auch dafür gibt es Gründe), ist mit den etablierten bibliometrischen Indikatoren keine qualitative Aussage und Bewertung möglich. Die überwiegende Anzahl der Wissenschaftler allerdings akzeptiert diese Annahmen zumindest in gewissen Grenzen, auch wenn unklar ist, in welcher mathematischen Beziehung die Anzahl der Zitierungen und die Qualität der Arbeit stehen.

Eine indirekte bibliometrische Analyse, wie sie die Resonanzanalyse darstellt, kann also immer nur Indizien für die Qualität einer Veröffentlichung liefern, niemals Beweise.

Eine weitere Einschränkung ergibt sich aus den verfügbaren Quellen, die herangezogen werden. Auch in den bekannten und (weltweit) anerkannten Datenbanken sind nicht alle Veröffentlichungen erfasst. Somit gibt es eine nicht unbeträchtliche Zahl von Publikationen, die eine bestimmte Arbeit zitieren, deren Zitierungen aber nicht mit in die Auswertung einfließen können, weil man über ihre Existenz nichts erfährt und sie nicht nachgewiesen sind. Diese Dunkelziffer ist nicht unerheblich. Allerdings hat sich, insbesondere in den Naturwissenschaften, der Medizin und den Ingenieurwissenschaften die Vorstellung durchgesetzt, dass die meisten relevanten 
Zitierungen in den etablierten Datenbanken nachgewiesen sind. Oder anders formuliert: Wenn eine wissenschaftliche Arbeit in einer Publikation zitiert wird, deren Bedeutung so gering ist, dass sie nicht in den relevanten Datenbanken ausgewertet wird, ist es auch nicht von Bedeutung, wenn diese Zitierung nicht gezählt wird.

Als Basisform der Resonanzanalyse kann der Grundindikator „Anzahl der Zitierungen“ vielfältig zu anderen (Performance-)Indikatoren in Beziehung gesetzt werden.

Die Zahl der Varianten ist dabei unüberschaubar, und täglich kommen neue Varianten in der Literatur hinzu. Dabei können auch sämtliche kritischen Bedenken eingebracht werden, etwa die Anzahl der Selbstzitierungen, die Berücksichtigung von Zitationszirkeln, die Publikationskultur der jeweiligen wissenschaftlichen Disziplin im Allgemeinen, die Anzahl der Autoren, die Position der Autoren in der Reihenfolge der Aufzählung, der zeitliche Abstand zwischen Erscheinen der Publikation und des Zitats, die Qualität der zitierenden Publikation und/oder des zitierenden Autors usw. usw.

\subsection{Rankings und Benchmarking}

Die Anwendung von bibliometrischen Analysen ergibt ihren größten Sinn nur im Vergleich, absolute Zahlen über den wissenschaftlichen Output und die Zitierhäufigkeiten ohne einen Benchmark sind nahezu ohne Aussage. Erst der direkte Vergleich macht Positionen sicht- und interpretierbar.

Doch die Nutzung von bibliometrischen Analysen zum Benchmarking ist eine der größten Herausforderungen der Bibliometrie. Denn um vergleichen zu können, müssen die Partner vergleichbar sein. Damit ist schon eine der wichtigsten Rahmenbedingungen für ein bibliometrisches Ranking beschrieben.

Es ist erforderlich, einen vergleichbaren Partner zu finden, das bedeutet etwa beim Ranking von Wissenschaftlern die Kenntnis darüber, in welchen Bereichen die Forscher jeweils arbeiten. Einen Biologen mit einem Chemiker direkt zu vergleichen, ist ebenso sinnlos wie einen experimentellen Physiker mit einem theoretischen. Bereits bei einem Vergleich in diesen stark verwandten Disziplinen sind die Publikationsgewohnheiten zu unterschiedlich. Nahezu unmöglich wird ein direkter Vergleich zwischen einem Germanisten und einem Mediziner oder einem Rechtswissenschaftler und einem Soziologen.

Allerdings haben die Statistiker in der Bibliometrie inzwischen auch Verfahren entwickelt, die einen transdisziplinären Vergleich ermöglichen. So etwa kann die relative Position von Wissenschaftlern oder Instituten innerhalb einer Fachcommunity bestimmt werden. ${ }^{5}$

5 Ball [u.a.] 2009: 381-392. 
Vergleichsanalysen sind daher stets eine Aufgabe für bibliometrische Spezialisten und erfordern neben der genauen Kenntnis der Rahmenbedingungen eine möglichst umfangreiche Zahlen- und Indikatorenbasis sowie die Anwendung möglichst verschiedener bibliometrischer Verfahren.

\subsection{Trendanalysen}

Bibliometrische Verfahren können auch eingesetzt werden, um wissenschaftliche oder inhaltliche Thementrends herauszufinden. In der thematischen Fokussierung und Konzentrierung von einschlägigen Publikationen zeigt sich bald, wo mögliche Trends abzulesen sind. Wenn bestimmte Themen über einen längeren Zeitraum auf hohem Niveau in (internationalen) Publikationen präsent sind, kann daraus ein Trendthema abgelesen werden. Umgekehrt zeigen nachlassende Zitierraten ein abnehmendes Interesse an dem Thema und damit einen Downtrend.

Es existieren allerdings auch sogenannte Sleeping Beauties, Themen also, die lange Zeit (zitationstechnisch) unbeachtet bleiben, ehe durch veränderte (meist externe) Rahmenbedingungen ein neues Interesse an diesen Themen entsteht. Dies beobachtet man häufig bei politisch beeinflussten oder gesteuerten Themen, etwa in der Umwelt- oder Energiepolitik. Wissenschaftliche Erkenntnisse zu einem bestimmten Thema werden dann schnell uninteressant, wenn sie politisch und damit auch finanziell nicht gefördert werden. Die Zitierhäufigkeiten solcher Arbeiten sind dann oft über Jahre sehr niedrig, was hier nicht an der Qualität der Publikationen liegt. Auch für high potentials, also überragende Wissenschaftler, existiert ein typischer Zitierverlauf: Nach der (üblichen) zeitlichen Verzögerung wird die Veröffentlichung auf hohem (nicht sehr hohem) Niveau zitiert, aber die Zitierhäufigkeit flaut über lange Jahre nicht ab.

Trendanalysen, die Vorhersagen zu wissenschaftlichen und gesellschaftsrelevanten Themen machen, werden insbesondere für strategische Entscheidungen von Wissenschaftsmanagern und -politikern genutzt. Damit kann Bibliometrie auch Unterstützung für den politischen Prozess der Themenfokussierung und der Etablierung neuer Forschungsförderungsgebiete samt neuer Infrastruktur leisten.

\section{Bibliometrische Indikatoren}

Ein bibliometrischer Indikator ist eine quantitativ erfass- und damit messbare Kennzahl, die eine Aussage zu einer wissenschaftlichen Veröffentlichung trifft. Die Anzahl von möglichen Indikatoren, die eine Aussage zu einer wissenschaftlichen Publikation machen, ist theoretisch unbegrenzt. Entscheidend für die Auswahl eines Indikators jedoch ist, welche Aussage denn getroffen werden soll. Somit determinieren Zweck 
und Zielsetzung einer bibliometrischen Analyse und damit die Fragestellung an eine wissenschaftliche Publikation den auszuwählenden, sinnvollen Indikator.

So kann man beispielsweise herausfinden wollen, wie oft die Publikation A von anderen zitiert worden ist. Der auszuwählende Indikator muss demnach eine Aussage treffen, in wie vielen anderen Publikationen die Veröffentlichung A zitiert worden ist. Dieser Indikator ist die Zitierhäufigkeit. Man könnte aber auch über die Publikation A wissen wollen, welche Wissenschaftler die Publikation A zitiert haben. Hier hilft die Zitierhäufigkeit nicht weiter, das Ergebnis dieser Frage kann nur eine Namensliste derjenigen Wissenschaftler sein, die die Publikation A zitiert haben. Will man etwa wissen, aus welchen Ländern diejenigen Publikationen kommen, in denen die Publikation A zitiert wird, ist das Ergebnis eine Länderliste. Auch weitergehende Fragestellungen an die Publikation A sind denkbar. So könnte man wissen wollen, aus welchen Fachdisziplinen diejenigen Wissenschaftler kommen, die Publikation A zitieren, wie lange sie schon in der Wissenschaft arbeiten, welche Bedeutung sie wiederum in der wissenschaftlichen Community haben usw. usw.

Zusammengefasst heißt dies: Ein bibliometrischer Indikator muss zunächst eine Antwort geben auf die konkrete Fragestellung, die an eine wissenschaftliche Veröffentlichung gerichtet ist. Zweitens muss der Indikator messbar sein, denn ein nur denkbarer, aber nicht zu erhebender Indikator ist sinnlos. Drittens muss der Indikator mit einer hohen Treffsicherheit und Qualität erhoben werden können, seine Werte dürfen also keine Zufallsergebnisse darstellen. Und viertens muss der Indikator mit einer vorher festzulegenden Aufwand/Nutzen-Korrelation zu erheben sein.

\subsection{Menge des wissenschaftlichen Outputs}

Die Basiskenngröße für eine bibliometrische Outputanalyse ist die Menge des wissenschaftlichen Outputs einer Person, einer Institution, eines Landes oder einer anderen (auf verschiedenen Ebenen aggregierten) Gruppe.

Dies schließt von vorneherein große Bereiche nicht-bibliometriegängiger Wissenschaftsleistungen aus. Die Anzahl wissenschaftlicher Veröffentlichungen ist nahezu die einzige bibliometrische Kennzahl, deren Erhebung von den relevanten und verfügbaren Datenbanken zur Bibliometrie unabhängig ist.

Denn die Anzahl von Veröffentlichungen kann man sehr einfach zählen, indem man alle verfügbaren Nachweisinstrumente thematischer und formaler Art (also Fachdatenbanken und bibliometrische Datenbanken) einbezieht sowie beliebige Internetquellen, gedruckte Quellen und Literaturlisten der Autoren selbst berücksichtigt und auswertet.

Damit kann man mit guter Näherung eine Vollständigkeit erzielen, die per se bei der Erhebung indirekter Indikatoren wie etwa der Zitationsraten nicht mehr möglich ist. 
Ein großer Aufwand ergibt sich bei der Erhebung der Anzahl wissenschaftlicher Veröffentlichungen durch den notwendigen Redundanzabgleich, der erforderlich ist, wenn viele verschiedene Quellen zur Bestimmung der Outputmenge herangezogen worden sind und damit Überschneidungen entstehen.

Im Ergebnis einer solchen Analyse entsteht eine Liste mit Publikationen. Die Abkürzung für die Anzahl der Paper ist N(p), was „number of papers“ bedeutet.

Die reine Anzahl der Publikationen ergibt jedoch noch keine wirklich brauchbare und qualifizierte Aussage über die Leistungsfähigkeit des Untersuchten.

Deshalb kann in einem zweiten Schritt eine Einteilung nach Publikationsarten erfolgen.

Diese Einteilung ist natürlich frei und damit auch beliebig, es haben sich jedoch in der Praxis einige berücksichtigte Standardformen durchgesetzt. So unterteilt man in Monographien (also Bücher), in Beiträge zu Büchern, Zeitschriftenaufsätze und Konferenzbeiträge in Sammelbänden. Darüber hinaus unterscheidet man zwischen der Autorenfunktion und der Herausgeberfunktion von Büchern und Sammelbänden.

In einem dritten Schritt kann man die Spezifika der Zeitschriftenbeiträge ausdifferenzieren. Dazu stellt man Fragen folgender Art: In welcher Art von Zeitschrift ist der Aufsatz publiziert worden, welche Bedeutung und Qualität hat die Zeitschrift (Impaktfaktor!), sind die Beiträge dort qualitätsgeprüft und einem peer review unterzogen usw.

Selbstverständlich kann man, noch ohne die Resonanzanalyse und ihre speziellen Indikatoren bemühen zu müssen, verschiedene weitere Untersuchungen mit den Ergebnissen der Outputanalyse anstellen. Wichtig jedoch ist allein, welche Sinnhaftigkeit die Fragen haben, die man an die Ergebnisliste und ihre einzelnen Einträge stellt. So kann man die Artikellänge analysieren (der Aussagewert ist sicher umstritten), die Sprache feststellen, in der die Artikel publiziert sind, oder die Länder der Zeitschriften feststellen, in denen publiziert wurde. Es könnte aber auch sinnvoll sein zu untersuchen, ob weitere Autoren an der Publikation mitgeschrieben haben (KoAutoren) und wie viele es sind. Darauf aufbauend kann man hinterfragen (und bibliometrisch herausfinden), aus welchen Instituten die Mitautoren kommen, aus welchen Ländern oder gar aus welchen (anderen) Wissenschaftsdisziplinen.

Daraus lässt sich dann - und dies bereits in diesem frühen Stadium einer einfachen Outputanalyse - erkennen, ob unser Autor oft mit weiteren Autoren zusammen publiziert, ob es ein Team ist, das (öfter?) zusammen arbeitet, oder ob eine offensichtliche wissenschaftliche Zusammenarbeit zwischen verschiedenen Institutionen existiert. Es ließe sich auch herausfinden und (womöglich) stichhaltig interpretieren, dass mit diesen Publikationen eine Interdisziplinarität verbunden ist, die so vielleicht vorher nicht gesehen wurde. Danach könnte man herauszufinden versuchen, welche verschiedenen Disziplinen hier an einem Forschungsthema zusammen arbeiten.

Es lassen sich auch zeitliche Verläufe aus der Publikationshäufigkeit ableiten: Man kann schauen, ob es Phasen intensiver Publikationstätigkeit gab und ableiten, 
welche Themen zu welchen Zeiten für das untersuchte Institut oder die untersuchte Person besonders wichtig waren.

Der Aussagewert einer Outputanalyse, also des ersten bibliometrischen Schritts, ist nicht so gering, wie er oft dargestellt wird. Natürlich kann die „nackte“ Zahl noch keine Aussage darüber treffen, wie gut oder schlecht ein Wissenschaftler oder ein Institut eigentlich ist, und auch ein Ranking auf Basis der reinen Outputzahlen sagt noch nichts aus.

Aber eine vertiefte Analyse, wie sie in den Beispielen oben geschildert worden ist, kann sehr wohl qualitative Aussagen über den Verlauf von Forschung, besondere Themenschwerpunkte oder wissenschaftliche (Länder- und Institutionen-übergreifende) Forschungskooperation ermöglichen.

\subsection{Zitierhäufigkeit, Zitierrate}

Mit den Kennzahlen „Zitierhäufigkeit“ und „Zitierrate“ beginnt die Komplexität der indirekten bibliometrischen Indikatoren, die sich nicht unmittelbar aus der Veröffentlichungsliste ableiten lassen und deren Wert nur über eine mittelbare Annahme Aussagefähigkeit erlangt.

Ist die Anzahl der Publikationen noch relativ leicht und im Zweifel ohne weitere Hilfsmittel $\mathrm{zu}$ eruieren, so entsteht bei der Messung der Zitierrate ein Bedarf an (maschineller) Massenauswertung. Denn die Zitierrate bestimmt die Anzahl der Zitate, die eine bestimmte Publikation erhalten hat. Dabei wird schon im Ansatz erkennbar, dass die Bestimmung der Zitierrate mit drei Unsicherheiten behaftet ist:

Erstens wird die erhobene Zahl der Zitierungen nie vollständig sein können, da es praktisch niemandem gelingt, sämtliche Publikationen weltweit nach Zitierungen der Ausgangspublikation zu untersuchen, und zweitens ist die Bestimmung der Anzahl der Zitierungen ein Massengeschäft, das händisch praktisch nicht realisierbar ist.

Und drittens müssen wir noch interpretieren (oder besser statistisch abschätzen), welche qualitative Aussage denn hinter der Zahl der Zitate steckt, die erhoben wird. Ohne Vorwissen (und dieses muss ganz gewaltig sein) können wir keine Aussage treffen, ob es viel oder wenig ist, wenn eine Publikation 10, 100, 1.000 oder 10.000 Mal zitiert worden ist.

Wieder einmal ergibt sich ein Sinn dieser Werte nur im Kontext anderer Zahlen, was bedeutet, dass Zitationszahlen immer relative Aussagen sind.

Der Grundindikator für die Anzahl der Zitationen ist N(C), also die number of citations.

Wie bereits beim Grundindikator N(P) kann man den Indikator N(C) in eine beliebige Zahl von Relationen setzen und interpretieren. In diesem Kapitel wollen wir die wichtigsten ansehen. 
Meist wird bei der Bestimmung der Zitierhäufigkeit nicht die absolute Zahl der Zitierungen gewählt, sondern die Anzahl der Zitate pro Veröffentlichung, die dann Zitierrate genannt und mit CPP abgekürzt wird. CPP bedeutet Citations per paper.

Man kann die Zitierrate, ähnlich wie die Anzahl der Zitate, für eine Person, eine Institution, eine Region oder ein Land berechnen.

Für einen konkreten Wissenschaftler etwa berechnet man die Zitierrate, indem man die Zahl aller Zitierungen, die die Publikationen des Wissenschaftlers erhalten haben, durch die Anzahl seiner Publikationen teilt, also $\mathrm{CPP}=\mathrm{N}(\mathrm{C}) / \mathrm{N}(\mathrm{P})$.

Ein Beispiel: Hat der Wissenschaftler im Zeitraum 1990 bis 201050 Veröffentlichungen geschrieben und 1.000 Zitierungen erhalten, so ergibt sich eine Zitierrate von $1.000 / 50=20$. Damit beträgt die Zitierrate des Wissenschaftlers im gesetzten Zeitraum 20.

Man kann die Anzahl der Zitierungen oder die Zitierrate in ganz beliebige Verhältnisse zu anderen Indikatoren der Veröffentlichungsumgebung setzen. Ein besonderer Effekt kommt jedoch bei der Resonanzanalyse hinzu: Hier kann man auf weiteren Ebenen die zitierenden Veröffentlichungen, also diejenigen Publikationen, die das Ausgangspaper zitiert haben, auf die verschiedensten Faktoren hin untersuchen. So ergibt sich eine Zitationsanalyse auf einer Metaebene (oder sogar auf den Metaebenen 1., 2. und x-ter Ordnung), indem man z.B. herausfindet, welche Wissenschaftler die Ausgangspublikation zitieren, in welchen Ländern sie arbeiten, welche Sprachen sie benutzen, welchen Disziplinen sie angehören etc.

Hieraus ergeben sich die verschiedensten (denkbaren) Kombinationsmöglichkeiten und neue Fragestellungen, die man sowohl an den Zitierenden als auch an das zitierende Paper stellen kann.

Immer wieder jedoch muss die Ausgangfrage konzis beantwortet sein, was denn eine Inbeziehungsetzung bedeutet und welche Aussage eigentlich getroffen wird. Mit Statistik lässt sich bekanntlich auch Unsinn vergleichen, und deshalb ist es erforderlich, dass zunächst geklärt ist, auf welche Frage man denn eine Antwort haben möchte oder (falls sich Antworten aus der Statistik ergeben) welche Aussage die erhaltenen Relationen eigentlich besitzen.

Oftmals zitieren sich Autoren in wissenschaftlichen Beiträgen selbst und verweisen auf andere, eigene Veröffentlichungen.

Deshalb wird bei bibliometrischen Resonanzanalysen oft der Anteil der Selbstoder Eigenzitierungen herausgerechnet, um Manipulationen vorzubeugen und Vergleichbarkeit zu erreichen. Gleichzeitig geht man davon aus, dass ein Fremdzitat einen größeren wissenschaftlichen Wert besitzt als ein Eigenzitat.

Wenn man Zitierungen im Verlaufe der Zeit analysiert, erhält man einen idealtypischen Verlauf. Etwa zwei Jahre nach der Veröffentlichung kann man mit der Berechnung der Zitierungen beginnen, da es eine ganze Weile dauert, bis der Beitrag in der jeweiligen Community angekommen und rezipiert worden ist. Die Zahl der Zitierungen steigt auf ein bestimmtes Niveau und fällt nach relativ kurzer Zeit (einige Monate bis wenige Jahre) wieder ab. Dieser normale Verlauf spiegelt damit den Lebenszyklus einer wissenschaftlichen Veröffentlichung wider. 
Die genaue Dauer der Phasen hängt stark von der Disziplin und den Themen ab.

Eine weitere Form der Resonanzanalyse ist die Verfolgung von Patenten und Patentzitaten. Patente werden oft ignoriert, da sie nicht für alle Disziplinen von Bedeutung sind und relevante bibliometrische Datenbanken Patente und Patentzitate nicht abbilden.

Tatsächlich jedoch sind in vielen wissenschaftlichen Disziplinen Patente als Forschungsoutput von großer Bedeutung und deren Zitierungen ein interessantes Maß für die Bedeutung der jeweiligen Patente bzw. ihrer Urheber. ${ }^{6}$

\subsection{Der Hirsch-Index}

Über lange Zeit wurde für die Beurteilung der wissenschaftlichen Leistungsfähigkeit einer Person primär die Zitierrate oder der Impaktfaktor eingesetzt. Erst vor wenigen Jahren wurde von dem amerikanischen Physiker Hirsch ein Indikator entwickelt, der eine genauere, objektivere und damit auch besser vergleichbare Bewertungsgrundlage für die wissenschaftliche Bedeutung von Einzelpersonen ermöglicht. ${ }^{7}$

Der Hirschfaktor ist, genau wie die Zitierrate, eine Kombination aus der Anzahl der Publikationen und der Zitierhäufigkeit.

Er wird ermittelt, indem alle Publikationen einer Person in der Reihenfolge ihrer Zitierhäufigkeit abnehmend sortiert werden. Derjenige Wert, bei dem die laufende Nummer der Publikation mit der Zierhäufigkeit übereinstimmt bezeichnet den „Hirsch-Faktor“.

Beispiel: ${ }^{8}$

Ein Wissenschaftler hat 8 Arbeiten publiziert. Listet man diese geordnet nach der Häufigkeit, wie oft sie zitiert worden sind, auf, kann man direkt ablesen, wie groß der Hirsch-Index ist:

Anzahl Zitationen

$\begin{array}{lr}1 & 32 \\ 2 & 25 \\ 3 & 21 \\ 4 & 13 \\ 5 & 7 \\ 6 & 5 \\ 7 & 4 \\ 8 & 1\end{array}$

5 Publikationen haben mindestens 5 Zitationen erlangt. Somit ist auch der Hirsch-Index des betreffenden Forschers 5.

6 Bartkowski 1999: 87-105.

7 Hirsch 2005.

8 Aus Hirsch 2005. 
Wie aus dem Beispiel zu erkennen ist, berücksichtigt der Hirschfaktor Publikationen mit unterdurchschnittlich geringer Zitierhäufigkeit und solche mit überdurchschnittlich hoher Zitierhäufigkeit weniger als durchschnittlich oft zitierte Paper. Das bedeutet, Extreme werden ausgeklammert zugunsten eines möglichst breiten Leistungsbilds.

Der Hirschindex wird seit seiner Einführung im Jahr 2005 sehr erfolgreich eingesetzt und ist inzwischen bei vielen Berufungsverfahren als Standard-Indikator etabliert. Es existiert inzwischen eine Vielzahl von Varianten des Hirsch-Faktors.

Als so genannter robuster Faktor, der Negativ- wie Positiv-Spitzen eliminiert, ist der Hirsch-Index als einfach zu berechnender und zu verstehender und zwischenzeitlich auch in allen relevanten bibliometrischen Datenbanken automatisiert zu generierender Indikator für die Beurteilung des Publikationsoutputs von Personen etabliert.

Die Abbildung 1 zeigt die H-Werte für Wissenschaftler verschiedener Disziplinen.

\begin{tabular}{llrr}
\hline Wissenschaftler & Fach & H-Index & Jahr \\
\hline Philip W. Anderson $^{9}$ & Physik & 91 & 2005 \\
George M. Whitesides $^{10}$ & Chemie & 169 & 2011 \\
Wolfgang Holzgreve $^{11}$ & Medizin & 41 & 2013 \\
Solomon H. Snyder $^{12}$ & Biologie & 191 & 2005 \\
\hline
\end{tabular}

Abb. 1: Werte für den H-Index ausgewählter Wissenschaftler verschiedener Disziplinen.

Es muss dennoch ausdrücklich erwähnt werden, dass es keinen Superindikator gibt, der - reduziert auf eine Zahl - ein objektives Bild für Output und Wahrnehmung wissenschaftlicher Publikationen liefern könnte. Auch der „Crown-Indikator“13 ergibt normalisiert über alle Fächer - lediglich eine überdisziplinäre Vergleichbarkeit der Kennzahlen, ebenso wie der am Forschungszentrum Jülich entwickelte J-Faktor. ${ }^{14}$

9 http://www.ncbi.nlm.nih.gov/pmc/articles/PMC2669244/ (31.03.2013).

$10 \mathrm{H}$-index ranking of living chemists, http://www.rsc.org/images/H-index $\% 20$ ranking $\% 200 f \% 20$ living\%20chemists(December\%202011)tcm18-211414.pdf. (31.03.2013).

11 http://www.wolfgang-holzgreve.de/curriculum-vitae/wolfgang-holzgreve-cv-deutsch/index.html (31.03.2013).

12 http://www.ncbi.nlm.nih.gov/pmc/articles/PMC2669244/ (31.03.2013).

13 „Der Zitatenjäger“ titelt „Die Zeit“ einen Beitrag aus 2003 über Anthony van Raan, einen der bedeutendsten Bibliometriker unserer Zeit. An der Universität Leiden erforscht van Raan in seinem Institut die bibliometrischen Zusammenhänge und erstellt Analysen und Forschungstrends. Spiewak 2003. Zum Crown Indicator: van Raan 2004.

14 Ball [u.a.] 2009: 381-392. 


\subsection{Der Impaktfaktor}

Die Zeitschrift als elementares Medium wissenschaftlicher Kommunikation ist eine der am häufigsten benutzten Quellen in bibliometrischen Untersuchungen. Der Impaktfaktor gilt als Maß zur Bewertung der Zeitschriftenqualität.

Verfolgt man die Anwendungshäufigkeit des Impaktfaktors und glaubt man den Wissenschaftlern, die ihn so gerne als eigenen Leistungsindex benutzen, ist der Impaktfaktor ein wahrer Wunderindex und Alleskönner. Er wird eingesetzt, um die Bedeutung und Qualität von Zeitschriften zu dokumentieren, und gleichzeitig ist der Impaktfaktor noch immer bei den meisten Berufungsverhandlungen von wissenschaftlichem Personal der Bewertungsmaßstab Nummer eins. Völlig zu Unrecht, wie man anhand der Analyse des Faktors leicht zeigen kann.

Der Impaktfaktor wird bestimmt durch die Anzahl der Artikel in einer Zeitschrift und der Anzahl der Zitate, die jeder Artikel bekommen hat. Dabei wird die Summe der Zitierungen durch die Zahl der Artikel geteilt. Da Zitierungen immer erst mit einer zeitlichen Verzögerung von rund zwei Jahren messbar sind, wird der Journal Impact Factor definiert als die Summe der Zitierungen geteilt durch alle Artikel einer Zeitschrift der vergangenen zwei Jahre.

Die Bestimmung und Veröffentlichung des Impaktfaktors erfolgt im Journal Citation Report. Dieser enthält nur eine begrenzte Auswahl an Zeitschriften und ignoriert bei der Auswertung Webpublikationen ebenso wie Monographien und graue Literatur. ${ }^{15}$ Es gibt deshalb eine ganze Reihe von Alternativen zum Impaktfaktor. ${ }^{16,17,18,19}$

Wie alle Querschnittsfaktoren (statistisches Mittel ) sagt der Impaktfaktor allerdings nichts über die Qualität einzelner Artikel aus: Es ist deshalb möglich, dass eine Zeitschrift ihren hohen Impaktfaktor und damit ihr gutes Image allein einigen wenigen, hoch zitierten Beiträgen verdankt, während die restlichen Artikel weniger oder kaum wahrgenommen werden.

Damit wird schnell klar, dass Personen nicht mit Hilfe des Impaktfaktors beurteilt werden können. Dennoch wird der Impaktfaktor, häufig als „kumulierter Impaktfaktor", noch immer als Maß für die wissenschaftliche Exzellenz von Wissenschaftlern missverwendet. ${ }^{20}$

Für die leistungsorientierte Mittelvergabe oder sogar für die Gewährung von Gehaltsboni bei Wissenschaftlern ist der Faktor und die eingesetzte Datengrundlage fragwürdig, da sie nicht transparent und damit nachvollziehbar gemacht wird. ${ }^{21}$

15 Sicilia [u.a.] 2011: 698-704.

16 Gonzáles-Pereira [u.a.] 2010: 379-391.

17 Leydesdorff [u.a.] 2011: 217-229.

18 Jacsó 2010: 339-348.

19 Zhang [u.a.] 2011: 643-653.

20 Für den kumulierten Impaktfaktor werden die Impakt-Faktoren mit der Anzahl ihrer Beiträge in den jeweiligen Zeitschriften multipliziert und in der Summe aufaddiert.

21 Rossner [u.a.] 2007. 


\begin{tabular}{llr}
\hline Disziplin & Zeitschrift & JIF (2011) \\
\hline Psychologie & Psychological Bulletin & 14,457 \\
Physik & Nature Materials & 32,841 \\
Genetik & Genomics & 3,019 \\
Chemie & Annual Review of Analytical Chemistry & 9,048 \\
Biologie & PLOS Biology & 11,452 \\
\hline
\end{tabular}

Abb. 2: Der Journal Impact Factor hängt sehr stark von der jeweiligen Wissenschaftsdisziplin ab. JIF ausgewählter Zeitschriften in unterschiedlichen Disziplinen (Quelle: JCR; Stand: 18.03.2013).

Der Journal Impact Factor hängt sehr stark von der jeweiligen Wissenschaftsdisziplin ab. Abbildung 2 zeigt Beispiele des JIF einiger ausgewählter Zeitschriften.

\subsection{Alternative Metriken}

Bibliometrische Analysen werden seit Jahrzehnten auf der Basis der bis dato einzigen verfügbaren Datenbank, des Science Citation Index (SCI) erstellt. Heute kommt zumindest ein kommerzielles Wettbewerbsprodukt SCOPUS hinzu. Zu diesem klassischen Prinzip der Zählung von Publikationen und der Nachverfolgung ihrer Zitierung in anderen Publikationen (zitationsbasierte Metriken) existieren heute Alternativen. ${ }^{22}$

Erst die massenhafte Verfügbarkeit von frei zugänglichen Publikationsdaten und gegebenenfalls ihrer Resonanzwerte hat in jüngster Zeit das Entstehen sogenannter alternativer Metriken oder abgekürzt „Altmetrics“ (aus dem englischen „alternative metrics") möglich gemacht. Da letztlich erst das Internet diese Art alternativer Metriken zulässt, spricht man hierbei auch von „Webmetrics“ oder in Anlehnung an den Begriff der Bibliometrie von „Webometrie“.

Systeme wie SCImago Journal Rank (SJR), Eigenfaktor oder der Index SourceNormalized Impact per Paper (SNIP) stellen heute noch keine wirklichen Alternativen dar, sind allerdings geeignet, das Denken über Kennzahlen und die dafür eingesetzte Datenbasis zu befruchten und zu erweitern. ${ }^{23,24,25}$

Ebenso werden zunehmend größere Mengen an relevanter wissenschaftlicher Information etwa in Form von Vorträgen und Präsentationen (Slideshare ${ }^{26}$ ) oder wissenschaftlicher Software (GitHub ${ }^{27}$ ) für die Wissenschaftsgemeinde frei im Netz zur Verfügung gestellt. Auch die Erfassung und Messung dieser Formen des wissen-

22 Bollen [u.a.] 2009.

$23 \mathrm{http}$ ://www.eigenfactor.org (31.03.2013).

24 West [u.a.] 2011.

25 SCImago 2007.

$26 \mathrm{http://de.slideshare.net} \mathrm{(31.03.2013).}$

27 https://github.com/ (31.03.2013). 
schaftlichen Outputs lassen Aussagen über Aktivität, Qualität und Quantität der Forschung eines Wissenschaftlers zu.

\subsection{Datenbanken für bibliometrische Analysen}

Bibliometrie steht und fällt mit der verfügbaren Datenbasis. Auch heute noch können vor allem speziellere Fragestellungen nicht auf Basis der verfügbaren (kommerziellen) Datenbanken beantwortet werden, und der Analyst muss sich die gewünschten Daten aus anderen Quellen beschaffen.

Dennoch verfügen wir heute über bibliometrische Datenbanken, die einen Großteil der für bibliometrische Analysen erforderlichen Fragestellungen beantworten lassen. Mehr noch: Durch eine konzise, transparente und professionelle Datenerfassung und Publikationsauswertung über einen langen Zeitraum stehen leistungsfähige Systeme zur Verfügung, die Bibliometrie auf einem hohen Niveau erst möglich gemacht haben.

Das Herzstück der heute als Verkaufsbündel konfektionierten multiplen Datenbank „Web of Knowledge“ ist nach wie vor der Science Citation Index. Er wertet im Jahr 2013 3.757 Zeitschriften aus 100 Disziplinen aus und beschränkt sich im Wesentlichen auf Naturwissenschaften, Medizin und Ingenieurwissenschaften. Der SCIExpanded verzeichnet im Jahr 2013 8.628 Zeitschriften aus 150 Fachgebieten.

Seit 1973 existiert auch ein Zitationsindex für die Sozialwissenschaften (Social Science Citation Index (SSCI)), seit 1978 der Arts and Humanities Citation Index (AHCI) für die Geisteswissenschaften.

Leider ist die Abdeckung in zeitlicher wie thematischer Hinsicht noch zu gering, um damit verlässliche Analysen durchzuführen.

Neben den genannten Citation Indices erstellt Thomson Reuters auch den Conference Proceedings Citation Index, der aus den ursprünglichen ISI Proceedings hervorgegangen ist und Konferenzbeiträge auswertet.

Der Journal Citation Report ist eine weitere bibliometrische Datenbank, die auf Basis der Zitierungen von Artikeln in einzelnen Zeitschriftentiteln den Journal Impact Factor ermittelt.

Im Jahr 2011 wurde der Book Citation Index etabliert: Er wertet wissenschaftliche Monographien aus.

Abbildung 3 zeigt die Abdeckung des SCI in verschiedenen Disziplinen über die Jahre. Deutlich wird dabei, dass mit Medizin, den Lebenswissenschaften und der Physik die zentralen STM-Themen abgedeckt sind.

Eine weitere Aufgliederung und Spezialisierung der bibliometrischen Datenbanken wird aktuell mit der Bildung regionaler Datenbanken, etwa dem Chinese Citation Index oder dem Arabic Citation Index, begonnen.

Im Web of Science (WoS) finden sich lediglich rund 5 Prozent der wissenschaftlichen Zeitschriften weltweit wieder. Publikationen in den restlichen 95 Prozent der 
weltweiten wissenschaftlichen Zeitschriften werden nicht berücksichtigt. Was aus Sicht der Redaktion des WoS ein Qualitätskriterium ist, bedeutet auf Seiten der Kritiker die Ignoranz eines Großteils des weltweiten wissenschaftlichen Outputs.

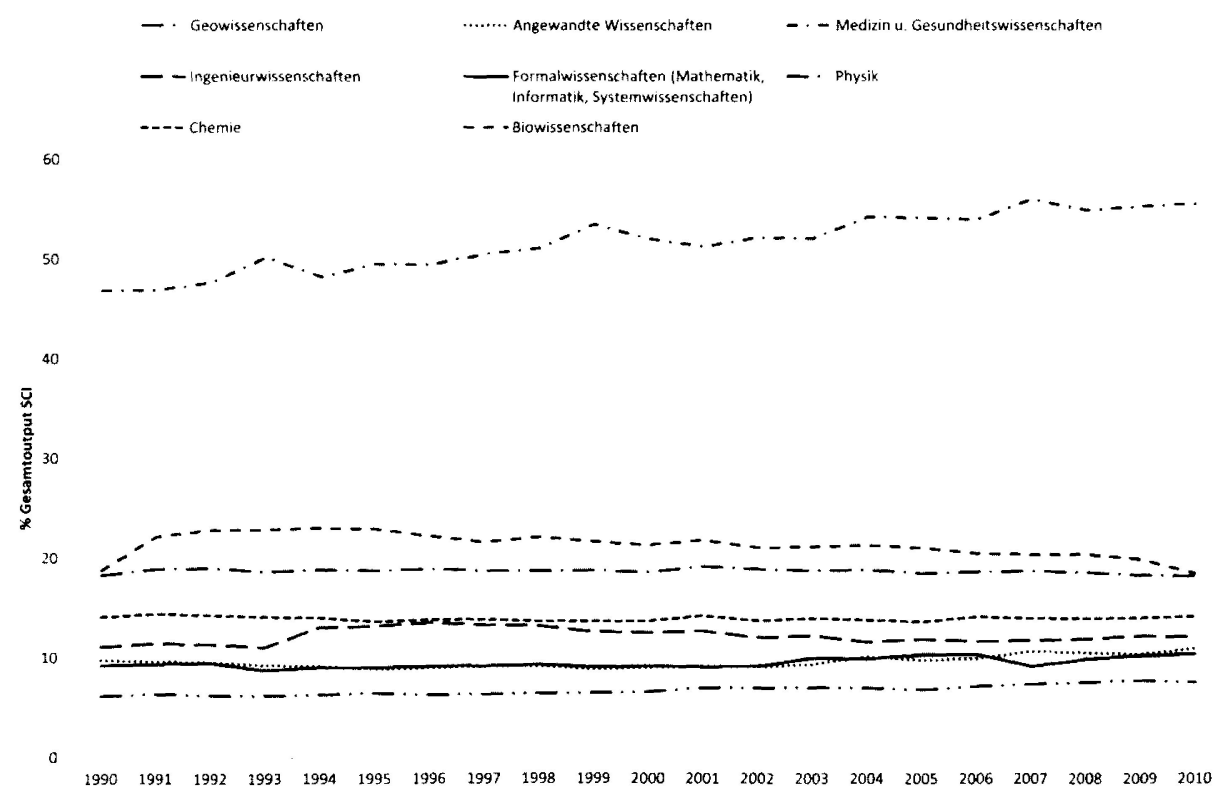

Abb. 3: Anteile wissenschaftlicher Publikationen verschiedener Disziplinen an der Gesamtanzahl der im SCl ausgewerteten Beiträge von 1990-2010.

Im Jahr 2004 begab sich Elsevier als internationales Medienunternehmen mit großer Erfahrung im wissenschaftlichen Publikationsgeschäft mit der eigenen Datenbank SCOPUS als Wettbewerber auf das komplexe Feld bibliometrischer Analysedatenbanken. ${ }^{28}$ SCOPUS verfolgt dabei einen anderen Ansatz als der SCI: Ein Gremium entscheidet über die Aufnahme von Zeitschriften und ihre Auswertung. Damit haben in dieser Datenbank auch Zeitschriften mit geringerem Impaktfaktor eine Chance, Wissenschaftler und Herausgeber können interessante Zeitschriften zur Aufnahme vorschlagen. Der große Wettbewerbsvorteil des SCI durch die langjährige Abdeckung hat sich relativ schnell aufgebraucht, Die Möglichkeiten der Massendigitalisierung erlaubten es SCOPUS, innerhalb sehr kurzer Zeit eine enorme Menge von Inhalten nachzuführen und eine zeitliche Abdeckung zu erreichen, die für belastbare bibliometrische Analysen schon ausreichend sind.

Damit verfügt der Markt über zwei kommerzielle Systeme, deren Datenerstellung und -nutzung auf einem sehr hohen automatisierten und technischen Niveau professionelle bibliometrische Analysen möglich machen.

28 http://www.scopus.com/ (31.03.2013). 
Häufig besteht der Wunsch, bibliometrische Analysen durchführen zu können, auch ohne auf „Bezahlinformation“ zurückgreifen zu müssen. Spätestens seit der Suchmaschinenriese Google seine Datenbankangebote um die Sparte Scholar erweiterte, schien die Community eine neue Option gefunden zu haben. Und tatsächlich spielte Google nicht nur wissenschaftliche Publikationen in das System ein, sondern auch Zitationsdaten. ${ }^{29}$ Es war also möglich, zumindest die Zahl Zitierungen einer Publikation zu finden; mehr noch, die Zitate sind meist verlinkt, und ein direkter Zugang zu den zitierenden Publikationen ist möglich. Dennoch stellt Google Scholar keine echte Alternative zu SCOPUS und dem SCI dar, da völlig unklar ist, welche Publikationen aufgenommen werden und wie die gemessenen Zitierungen zu Stande kommen.

\section{Bibliometrische Analysen als Geschäftsfeld für Bibliotheken}

Die Rahmenbedingungen für die bibliothekarische Arbeit haben sich in den letzten Jahrzehnten grundlegend verändert. Bibliotheken sämtlicher Typen machen eine neue Standortbestimmung durch, in deren Verlauf die traditionellen Aufgabenfelder zunehmend in Frage gestellt und durch neue ergänzt werden. Es ist damit wichtiger denn je, die Produkte der Bibliotheken unter die Lupe zu nehmen und sie in einer Portfolioanalyse hinsichtlich ihrer Bedeutung zu überprüfen. ${ }^{30}$

Bei der Identifizierung neuer Handlungsfelder muss allerdings die Frage nach Konzentration auf Kernkompetenzen vs. Diversifizierung des Dienstleistungs-Portfolios gestellt werden dürfen. ${ }^{31}$

Informations- und Bibliothekswissenschaftler haben das Thema Bibliometrie Ende der 1990er Jahre vor dem Hintergrund einer veränderten Wissenschaftslandschaft auch für Bibliotheken sichtbar werden lassen. Große Mengen digital verfügbarer bibliometrischer Daten, die nun leicht prozessierbar waren, sowie die Notwendigkeit belastbarer quantifizierbarer Aussagen zu wissenschaftlichen Leistungen und die vielfache Einführung von leistungsorientierter Mittelvergabe in Wissenschaft und Forschung ${ }^{32}$ haben Fragen nach den Einsatzmöglichkeiten der Bibliometrie wieder zu einem aktu-

$29 \mathrm{http://scholar.google.de/(31.03.2013).}$

30 Ball 2000.

31 Ball 1999: 11-22.

32 „In der leistungsorientierten Mittelvergabe (LOM) sieht der Wissenschaftsrat ein zentrales Instrument der Forschungs- und Lehrförderung, das sowohl innerhalb der Fakultäten als auch auf der Ebene eines Bundeslandes zum Einsatz kommen sollte [...] Ziel ist, Leistungsträger zu belohnen und Anreize zu setzen, Leistungen in Forschung und Lehre zu steigern." Zur Weiterentwicklung dieser Instrumente hat der Wissenschaftsrat in der Standortübergreifenden Stellungnahme zur Weiterentwicklung der Universitätsmedizin in Baden-Württemberg (2004) Leitlinien erarbeitet. 
ellen Thema werden lassen. ${ }^{33}$ So erlebte die Bibliometrie einen zweiten Frühling, aber vornehmlich nicht in ihrer mathematischen Modellierung und theoretischen Grundlegung, sondern als Instrument im Wissenschaftsmanagement.

Völlig unklar hingegen ist die Frage, wer über die Kompetenz zur Durchführung bibliometrischer Analysen verfügt. Einige wenige sozial- und politikwissenschaftliche Institute versuchen sich an Bibliometrie, häufig jedoch nur auf der Metaebene, als Wissenschaft von der Bibliometrie. Die Informationswissenschaftler selbst haben dieses Feld ebenfalls nicht aktiv besetzt. So ist es keine Seltenheit, dass Assistenten und Sekretariate von Wissenschaftsmanagern mit derartigen Aufgaben betraut werden, womit sie natürlich genauso überfordert sind wie die Wissenschaftler selbst, deren Kompetenz zur Nutzung von elektronischen Informationsquellen noch immer zu wünschen übrig lässt. ${ }^{34}$

Informationsspezialisten stehen heute im Zentrum von gewaltigen Datenmengen, die über Wissenschaft und ihren Output weltweit zur Verfügung stehen. Sie sind als Informationsspezialisten grundsätzlich in der Lage, mit diesen Datenmengen umzugehen und aus ihnen fundierte, belastbare Informationen herauszukristallisieren. Wer sonst im wissenschaftlichen Umfeld könnte und sollte bibliometrische Daten als Service für Wissenschaftsmanager liefern - interdisziplinär und unabhängig von wissenschaftlichem Eigeninteresse? Nur Bibliotheken und Informationseinrichtungen sind unabhängige, interdisziplinäre Institutionen, die diese zentralen Dienste leisten können.

\section{Literatur}

Abbot, Andrew: Varianten der Unwissenheit. In: Nach Feierabend. Hrsg. von David Guferli, Michael Hagner, Philipp Sarasin u. Jakob Tanner. Zürich: diaphanes 2010 (Zürcher Jahrbuch für Wissensgeschichte 6). S. 15-34.

Almind, Tomas C. u. Peter Ingwersen: Informetric analyses on the World Wide Web. Methodological approaches to ,webometrics'. In: Journal of Documentation 53 (1997), H. 4, S. 404-426.

Ball, Rafael: Die Diversifizierung von Bibliotheksdienstleistungen als Überlebensstrategie. In: B.I.T. online 2 (1999), H. 1, S. 11-22.

Ball, Rafael: Die Cash Cow im Dienstleistungsportfolio einer Bibliothek oder: was macht Bibliotheken heute noch attraktiv? In: Vorträge zum 26. Österreichischen Bibliothekartag, Universitätscampus Wien, 19.-23. September 2000. Hrsg. vom VÖB. Internet-Publikation. Wien: 2000. S. 1-12.

Ball, Rafael, Bernhard Mittermaier u. Dirk Tunger: Creation of journal-based publication profiles of scientific institutions. A methodology for the interdisciplinary comparison of scientific research based on the J-factor. In: Scientometrics 81 (2009), H. 2, S. 381-392.

Ball, Rafael, M. Stein-Arsic, B. Buscher U. E. Bickar: Bibliometrische Analysen als Instrument des Bestandsmanagements in Bibliotheken. In: B.I.T. online 6 (2003), H. 4, S. 347-351.

33 Ball [u.a.] 2003: 347-351.

34 Klatt [u.a.] 2001. 
Ball, Rafael u. Dirk Tunger: Bibliometrische Analysen - Daten, Fakten und Methoden. Grundwissen Bibliometrie für Wissenschaftler, Wissenschaftsmanager, Forschungseinrichtungen und Hochschulen. Jülich 2005.

Bartkowski, Adam: Ausnutzung der Online-Datenbanken für die bibliometrische Bewertung neuer technischer Lösungen für Lizenzzwecke. In: PATINFO (1999). 21. Kolloquium der Technischen Universität Ilmenau über Patentinformation. Patent- und Markeninformation - Internet und Mehrwertdienste 10. und 11. Juni 1999. S. 87-105.

Bollen, Johan, Herbert Van De Sompel, Aric Hagberg u. Ryan Chute: A principal component analysis of 39 scientific impact measures. In: PLOS ONE (2009) 4 (6). e6022. doi:10.1371/journal. pone.0006022.

Bradford, Samuel: Sources of Information on Specific Subjects. In: Engineering 137 (1934), S. 85-86.

Garfield, Eugene: Citation Indexes for Science. A New Dimension in Documentation through Association of Ideas. In: Science 122 (1955), H. 3159, S. 108-111.

Gonzáles-Pereira, Borja, Vicente P. Guerrero-Bote $u$. Félix Moya-Anegón: A new approach to the metric of journals' scientific prestige: The SJR indicator. In: Journal of Informetrics 4 (2010), H. 3, S. 379-391.

Gorraiz, Juan: Szientometrie: Zitatenanalyse. Fachhochschulstudiengang Informationsberufe, Skriptum für Informationswissenschaft und -theorie, Teil 2. 2004. 23 S.

Gross, Paul L. K. U. Elsie M. Gross: College libraries and chemical education. In: Science 66 (1927), H. 1713 , S. 385-389.

Hirsch, Jorge E.: An index to quantify an individual's scientific research output. In: PNAS 102 (2005), H. 46, S. $16569-16572$.

Jacsó, Péter: Eigenfactor and article influence scores in the Journal Citation Reports. In: Online Information Review 34 (2010), H. 2, S. 339-348.

Klatt, Rüdiger, Konstantin Gavriilidis, Kirsten Kleinsimlinghaus u. Maresa Feldmann: Nutzung elektronischer wissenschaftlicher Information in der Hochschulausbildung. Barrieren und Potenziale der innovativen Mediennutzung im Lernalltag der Hochschulen. Dortmund 2001.

Leydesdorff, Loet u. Lutz Bornmann: How Fractional Counting of Citations Affects the Impact Factor: Normalization in Terms of Differences in Citation Potentials Among Fields of Science. In: Journal of the American Society for Information Science and Technology 62 (2011), H. 2, S. 217-229.

Lotka, Alfred J.: The frequency distribution of scientific productivity. In: Journal of the Washington Academy of Sciences 16 (1926), S. 317-323.

Mabe, Michael u. Mayur Amin: Growth dynamics of scholarly and scientific journals. In: Scientometrics 51 (2001), H. 1, S. 147-162.

Moed, H. F., R. E. De Bruin, A. J. Nederhof, A. F. J. Van Raan U. R. J. W. Tijssen: State of the art of bibliometric macro-indicators. An overview of demand and supply. In: Science 149 (1992), S. 510-515.

Price, Derek J. de Solla: Networks of scientific papers. In: Science (1965), Bd. 149, S. 510-515.

Price, Derek J. de Solla: Little Science, Big Science. Von der Studierstube zur Großforschung. Frankfurt a.M.: Suhrkamp 1974.

Pritchard, Alan: Statistical bibliography or bibliometrics? In: Journal of Documentation 25 (1969), H. 4, S. 348-349.

Raan, Anthony F. J. van: Measuring science. Capita selecta of current main issues. In: Handbook of quantitative science and technology research. The use of publication and patent statistics in studies of S\&T systems. Hrsg. von Henk F. Moed, Wolfgang Glänzel u. Ulrich Schmoch. Dordrecht: Kluwer Academic Publishers 2004. S. 19-51.

Rossner, Mike, Heather Van Epps u. Emma Hill: Show me the date. In: The Journal of cell biology 179 (2007), H. 6, S. 1091 f. 
Sicilia, Miguel-Angel, Salvador Sánchez-Alonso u. Elena García-Barriocanal: Comparing impact factors from two different citation databases: The case of Computer Science. In: Journal of Informetrics 5 (2011), H. 4, S. 698-704.

Spiewak, Martin: Der Zitatenjäger. Kann man die Qualität von Wissenschaft messen? Anthony van Raan arbeitet daran. In: Die Zeit 29/2003.

Taubert, Niels. C.: Bibliometrie in der Forschungsevaluation. Zur Konstitution und Funktionslogik wechselseitiger Beobachtung zwischen Wissenschaft und Politik. In: Quoten, Kurven und Profile. Zur Vermessung der sozialen Welt. Hrsg. von Jan-Hendrik Passoth u. Josef Wehner. Wiesbaden: Springer 2013. S. 179-204.

Thelwall, Mike, Antje Klitkou, Arnold Verbeek, David Stuart u. Celine Vincent: Policy-Relevant Webometrics for Individual Scientific Fields. In: Journal of the American Society for Information Science and Technology 61 (2010), H. 7, S. 1464-1475.

Thelwall, Mike u. Pardeep Sud: A Comparison of Methods for Collecting Web Citation Data for Academic Organizations. In: Journal of the American Society for Information Science and Technology 62 (2011), H. 8, S. 1488-1497.

Vickery, Brian C.: Bradford's Law of Scattering. In: Journal of Documentation 4 (1948), S. 198-203. West, Jevin D., Michael C. Jensen, Ralph J. Dandrea, Gregory J. Gordon u. Carl T. Bergstrom: Author-Level Eigenfactor Metrics: Evaluating the Influence of Authors, Institutions and Countries Within the SSRN Community. In: Journal of the American Society for Information Science and Technology 64 (2013), H. 4, S. 787-801.

Wouters, Paul u. Rodrigo Costas: Users, narcissism and control - tracking the impact of scholarly publications in the 21st century. http://www.surf.nl/nl/publicaties/Documents/Users $\% 20$ narcissism\%20and\%20control.pdf (31.03.2013).

Zhang, Cheng, Xu Liu, Yunjie (Calvin) Xu u. Youwei Wang: Quality-Structure Index: A New Metric to Measure Scientific Journal Influence. In: Journal of the American Society for Information Science and Technology 62 (2011), H. 4, S. 643-653. doi: 10.1002/asi.21487.

\section{Weiterführende Literatur}

Adams, David: The counting house. In: Nature 415 (2002), S. 726-729.

Ball, Rafael: Wissenschaft und Bibliotheken: das aktive Engagement im Kontext elektronischen Publizierens. 2000 (Zeitschrift für Bibliothekswesen und Bibliographie - Sonderhefte 80). S. 21-36.

Ball, Rafael: Der Hirschfaktor. Ein neuer „Stern“ am bibliometrischen Indikatorenhimmel. In: B.I.T. online 9 (2006), H. 4, S. 309-312.

Bellis, Nicola de: Bibliometrics and citation analysis: from the Science citation index to cybermetrics. Lanham, Md.: Scarecrow Press 2009.

Bergstrom, Carl T.: Eigenfactor: Measuring the value and prestige of scholarly journals. In: College \& Research Libraries News 68 (2007), H. 5, S. 314-316.

Björneborn, L. U. P. Ingwersen: Perspective of webometrics. Scientometrics 50 (2001), H. 1, S. 65-82. Borgman, C. L.: Bibliometrics and Scholarly Communication. In: Communication Research 16 (1989), H. 5, S. 583-599.

Bradford, Samuel: Sources of Information on Specific Subjects. In: Engineering 137 (1934), S. 85-86. Cole, Francis J. u. Nelli B. Eales: A history of comparative anatomy. Part I: A statistical analysis of the literature. In: Science Progress 11 (1917), S. 578-596. 
Garfield, Eugene: Is there a future for the scientific journal? Essays of an Information Scientist, 2 (1974-1976), S. 318-322. Current contents 31 (1975), S. 5-9. Reprinted from: Sci-Tech News, 29 (1975), H. 2, S. 42-44.

Garfield, Eugene: Is Citation Analysis a Legitimate Evaluation Tool? In: Evaluations of Individual Scientists and Research Institutions. A selection of papers reprinted from the journal Scientometrics. Hrsg. von T. Braun. Budapest: Akadémiai Kiadó Zrt (Scientometrics Guidebooks Series, Vol. 1). S. 955-971. Erstmals erschienen in: Scientometrics 1 (1979), H. 4, S. 359-375.

Hartling, Florian: Der digitale Autor. Autorschaft im Zeitalter des Internets. Bielefeld: Transcript Verlag 2009.

Kaube, f.: Forscher, was ist dein Wert. In: Frankfurter Allgemeine Sonntagszeitung (2006), Nr. 39, 01.10.2006, S. 82.

Lawrence, P. A.: The politics of publication. In: Nature 422 (2003), S. 259-261.

Lotka, Alfred J.: The frequency distribution of scientific productivity. In: Journal of the Washington Academy of Sciences 16 (1926), S. 317-323.

Marchi, M. de U. M. Rocchi: Editorial policies of scientific journals: testing an impact factor model. In: Scientometrics 51 (2001), H. 2, S. 395-404.

Melin, G.: Impact of national size on research collaboration. In: Scientometrics 46 (1999), H. 1, S. 161-170.

Parthey, Heinrich: Bibliometrische Profile wissenschaftlicher Institutionen in Problemfeldern und Phasen der Forschung. In: Evaluation wissenschaftlicher Institutionen: Wissenschaftsforschung Jahrbuch 2003. Hrsg. von Heinrich Parthey u. Heinrich Fischer. Berlin: Gesellschaft für Wissenschaftsforschung 2004. S. 63-102.

Poynder, R.: Are Reed Elsevier and Thomson Corp. monopolists? In: Information Today 18 (2001), H. 6, S. 1-58.

Vinkler, Péter: The evaluation of research by scientometric indicators. Oxford [u.a.]: Chandos Publishing 2010.

Weingart, Peter: Verführerische Zahlen. Die Messung der Forschung und ihre ungewollten Folgen. In: Neue Zürcher Zeitung, 13. April 2004, S. 11. 